\title{
The Interplay and Synergism of Experiment and Quantum Chemistry in Research on Reactive Intermediates
}

\author{
Thomas Bally*
}

\begin{abstract}
Three examples from the work of the author's research group on reactive intermediates are used to illustrate how fruitful interaction between experimental and computational chemistry may lead to the solution of problems that neither of the two methods could have solved without the other. It is shown that computational chemistry has indeed become an indispensable tool for the verification of spectroscopic assignments, the elucidation of reaction mechanisms, and the understanding of the factors that drive chemistry.
\end{abstract}

Keywords: Computational chemistry · Matrix isolation spectroscopy · Reactive intermediates

\section{Introduction}

The ability to predict and control the outcome of chemical and biological reactions frequently requires knowledge of the key reaction intermediates. However, such species are usually very labile, so their properties cannot be studied under normal conditions. To overcome this impediment, one can basically choose between two experimental approaches: (a) generate reactive intermediates as fleeting species in solution by some pulsed method (laser flash photolysis or pulse radiolysis) and follow their disappearance, or the appearance of secondary products, by a time-resolved form of spectroscopy

\footnotetext{
${ }^{\star}$ Correspondence: Prof. Dr. T. Bally

Département de Chimie

Université de Fribourg

Chemin du Musée 9

$\mathrm{CH}-1700$ Fribourg

Tel.: +4126300 8705

Fax: +4126300 9737

E-Mail: Thomas.Bally@unifr.ch
}

or (b) generate reactive intermediates under conditions where they persist during the time of the experiment, e.g. under collision-free conditions in the gas phase or by enclosing them in a solid, inert matrix, and probing them more or less leisurely by conventional techniques.

The two approaches often yield complementary information: time-resolved methods give insight into the kinetics of the decay of reactive intermediates, which is very important to understand their role in a chemical reaction, but they usually do not provide spectra containing much detailed information. Conversely, stabilized reactive intermediates may be subjected to higher resolution spectroscopies which yield information about the molecular and electronic structure, but this insight is gained at the loss of the kinetic information. On the other hand, the stabilization of reactive intermediates also allows them to be subjected to photoexcitation which induces rearrangements or fragmentations typical of such species, or which may lead to new compounds that are interesting in their own right.

For the past 20 years my research group has been carrying out a program of investigating organic reactive intermediates (neutral and charged radicals, carbenes, nitrenes, and others) by generating such species in noble gas matrices where they are often indefinitely stable because they are isolated in a medium that prevents diffusion and is kept at a temperature (typically $6-15 \mathrm{~K}$ ) at which a barrier of only $3-4 \mathrm{~kJ} / \mathrm{mol}$ prevents a unimolecular rearrangement or fragmentations for many hours [1]. Thus it is possible to probe these species in detail and to provide spectroscopic information which can subsequently be used in timeresolved experiments to identify them.

However, we are often confronted with the situation that a targeted species is formed in a mixture with rearranged or fragmented products, or that photolysis or controlled annealing of the matrix leads to new, unknown species. Then the problem arises how to make sense of the observed spectra and, once an assignment has been achieved, how to understand the mechanisms by which the observed chemical changes have taken place. Finally, the spectra often contain much valuable information on the molecular and electronic structure of the signal carriers, but this information is not always readily accessible.

This is where computational chemistry steps in, and, with the advent of modern workstations in the early 1990s, this part of science has indeed turned into an indispensable branch of our research program. The synergetic application of experiment and computational quantum chemistry has allowed us to address and solve many problems (often in collaboration with colleagues with complementary expertise). Thus we have pinned down products and reaction mechanisms whose occurrence calculations alone would not have predicted and experiment alone would not have been able to identify. However, the path to such successes is often a sinuous 
one. Following it has taught us a number of interesting lessons, both about the chemistry of reactive intermediates and the scope and limitations of some theoretical models.

This brief account shall illustrate the role that computational chemistry plays in our research on a few examples drawn from published and unpublished recent work.

\section{Napthylcarbomethoxycarbene}

A typical case where an expected reactive intermediate underwent a photoinduced rearrangement to an unknown secondary species was that of the carbene 1 which has a triplet ground state with a characteristic pattern of UV/Vis absorption bands [2]. This rearrangement, which was reversible both photochemically (at $515 \mathrm{~nm}$ ) and thermally (at $12 \mathrm{~K}$ !), gave difference spectra in the UV/Vis and IR range (Fig. 1) that showed the rise and decay of the new, unknown product $\mathbf{X}$. To assign a structure to $\mathbf{X}$ we calculated the UV/Vis transitions (by the semiempirical INDO/S method) and the IR spectra (by the B3LYP density functional method) of different isomers of $\mathbf{1}$ which could conceivably be expected to show the behavior documented in Fig. 1.

First we considered the known products of the parent naphthylcarbene, viz. cyclopropenes 2 or $\mathbf{3}$ (Scheme 1). However, these species were predicted to show intense IR bands for a $\mathrm{C}=\mathrm{C}$ stretching vibration above $1800 \mathrm{~cm}^{-1}$ and a $\mathrm{C}=\mathrm{O}$ stretch at $c a .1730 \mathrm{~cm}^{-1}$, a region where the experimental IR spectra show no changes. Apart from that $\mathbf{2}$ was not expected to absorb above $300 \mathrm{~nm}$ and $\mathbf{3}$ was calculated to lie significantly above ${ }^{3} \mathbf{1}$ in energy. Hence this straightforward assignment had to be discarded. Next we considered the possibility of a reversible rearrangement $\mathbf{1} \rightarrow \mathbf{5}$, possibly involving a metastable oxirene $\mathbf{4}$, but the barrier for this rearrangement was predicted to be prohibitively high, and the UV/Vis and IR spectra calculated for 5 showed no relationship to the observed spectra. In contrast, we did observe traces of ketene 6 that is formed by Wolff-rearrangement (or 1,2 acyl shift) from $\mathbf{3}$ or $\mathbf{5}$ (Scheme 1).

The above rearrangements take place on the singlet potential surface, so we also calculated the singlet state of the carbene ${ }^{1} \mathbf{1}$ which lies slightly higher in energy than ${ }^{3} 1$ but differs from the latter by the conformation of the carbomethoxy group which is coplanar with the naphthylcarbene moiety in ${ }^{3} \mathbf{1}$ but perpendicular to it in ${ }^{1} \mathbf{1}$ (Scheme 2):
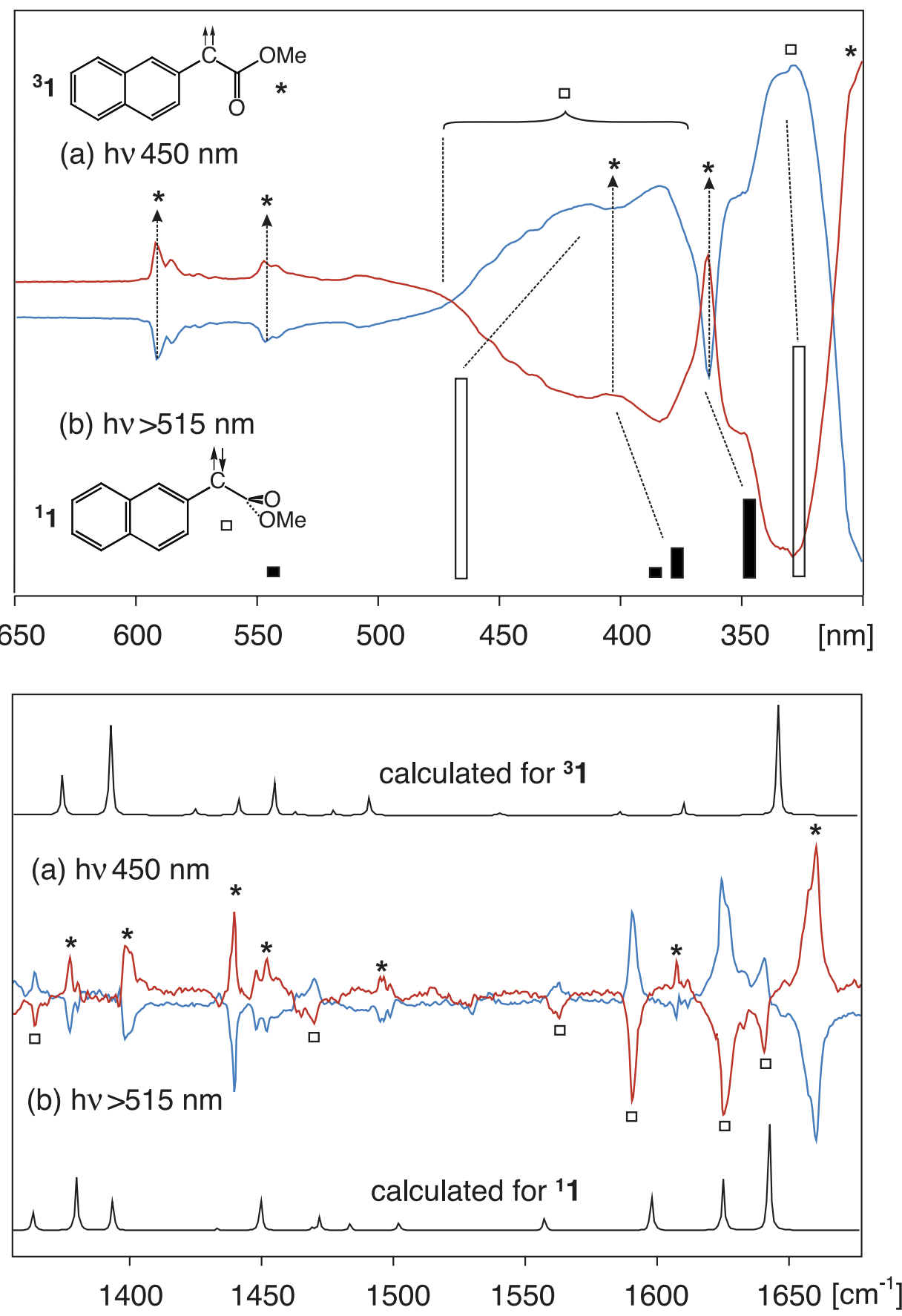

Fig. 1. Changes in the UV/Vis and IR spectra on bleaching of triplet naphthyl carbomethoxycarbene ${ }^{3} \mathbf{1}$ (blue lines), and its re-formation from the singlet state of the same carbene, ${ }^{1} \mathbf{1}$ (red lines). The solid bars under the UV/Vis spectra indicate excited state energies and transition moments from INDO/S calculations for ${ }^{3} \mathbf{1}$, the open bars for ${ }^{1} \mathbf{1}$. The theoretical IR spectra were calculated by B3LYP/6-32G* and all frequencies were scaled by 0.97 .

The graduate student in charge of this project [2] found that the calculated IR and UV/Vis spectra of ${ }^{1} \mathbf{1}$ are actually in good agreement with those observed for the enigmatic photoproduct of ${ }^{3} \mathbf{1}$ (Fig. 1) and thus proceeded to propose that what he had seen was a reversible interconversion between two stable spin states of the same carbene.

This proposal turned out to be hard to defend because such 'spin isomerism' had never been observed before in organic systems. With the help of Prof. McMahon from the University of Wisconsin, who shared our fascination for this intriguing system and provided ESR measurements that showed the rise and decay of a triplet, we eventually convinced the very skeptical reviewers and got our results and their interpretation published [2]. This paper was followed up by some time-resolved studies which determined the kinetics and the thermochemistry of the interconversion between ${ }^{1} \mathbf{1}$ and ${ }^{3} \mathbf{1}$ which to date stands as the only documented example of 'spin isomerism' in organic chemistry [3][4]. 


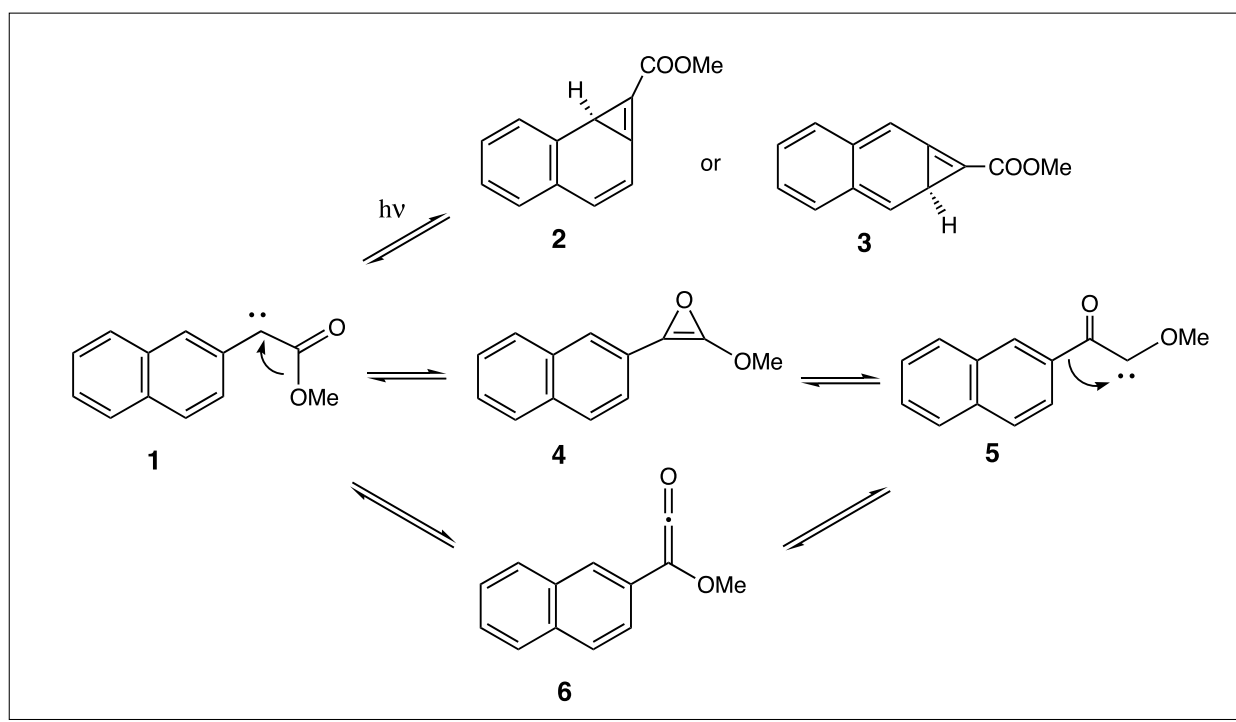

Scheme 1

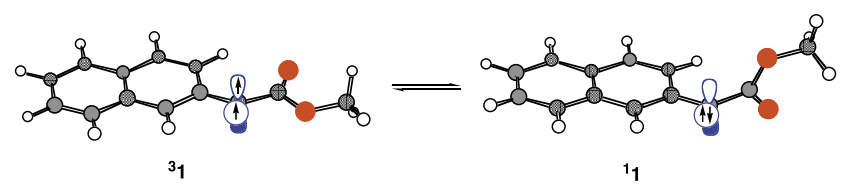

Scheme 2 .

This example shows that the match between calculated and observed IR or UV/ Vis transitions does not need to be quantitative to gain confidence in an assignment, although it is crucial to ascertain that no intense predicted bands are missing from the experimental and no intense observed bands from the calculated spectrum. Also, one must realize that in IR difference spectra intense rising bands may mask weaker decreasing ones, which seems to be what happens at 1390 and $1450 \mathrm{~cm}^{-1}$ in the present case. In spite of this, the presence of three bands between 1590 and $1650 \mathrm{~cm}^{-1}$ (together with the absence of bands above $1650 \mathrm{~cm}^{-1}$ ) proved characteristic of the structure of ${ }^{1} \mathbf{1}$. Fortunately, with the advent of hybrid density functional methods, we have a computational tool at our disposition which allows the prediction of IR spectra with an accuracy that is usually sufficient for the identification of unknown compounds up to medium size ( $\mathbf{1}$ is at the upper limit) by their IR band patterns, perhaps after scaling all frequencies with a single factor.

In the above case, and at the time when this study was carried out, it was inconceivable to perform excited state calculations on systems the size of $\mathbf{1}$ with anything but semiempirical methods (which proved to be sufficiently accurate in this case to support the proposed assignments). However, semiempirical electronic structure models have their limitations (e.g. they generally underestimate through-space interactions of $\pi$-systems and lone pairs). In the 1990s, the group of Roos has provided us with an $a b$ initio method that allows to account adequately for the nondynamic (configuration mixing) effects that often prevail in excited states as well as for the pronounced variations in dynamic electron correlation between different electronic states and thus allows reasonably accurate predictions to be made of electronic spectra. An illustration for the application of this so-called CASSCF/CASPT2 procedure [5] in the elucidation of the structure of reactive intermediates will be given below.

\section{The Radical Cation of syn-Tricyclooctadiene [6]}

Upon ionization of the syn-tricyclooctadiene 7 by pulse radiolysis in an organic glass at $30 \mathrm{~K}$, one observes a very strong band at $540 \mathrm{~nm}$, accompanied by a shoulder at $505 \mathrm{~nm}$ that is due to the radical cation of cyclooctatetraene $\left(\mathrm{COT}^{\circ+}\right)$ [7], a 'logical' rearrangement product of $\mathbf{7}^{\circ}$. In addition one finds a weaker band at $c a .820 \mathrm{~nm}$ which grows at the expense of the $450 \mathrm{~nm}$ band on warming the matrix to $75 \mathrm{~K}$ and disappears in turn on heating above $90 \mathrm{~K}$,
Fig. 2. (a): Spectrum obtained after ionization of a $0.01 \mathrm{M}$ glassy solution of the syn-tricyclooctadiene 7 in methylclohexane containing $1 \mathrm{M} \mathrm{n}$-butyl chloride by pulse radiolysis at $30 \mathrm{~K}$; (b) and (c): after annealing the matrix at $75 \mathrm{~K}$ for 65 and $215 \mathrm{~min}$, respectively; (d) after prolonged annealing of the matrix at $90 \mathrm{~K}$. The dashed line represents the spectrum of the radical cation of cyclooctatatetraene $\left(\mathrm{COT}^{+\bullet}\right)$. Spectrum $B$ is the spectrum of the species which grows on annealing at $75 \mathrm{~K}$ and decays on annealing at $90 \mathrm{~K}$ and spectrum $\mathrm{C}$ is that of the product which grows on decay of $\mathrm{B}$.

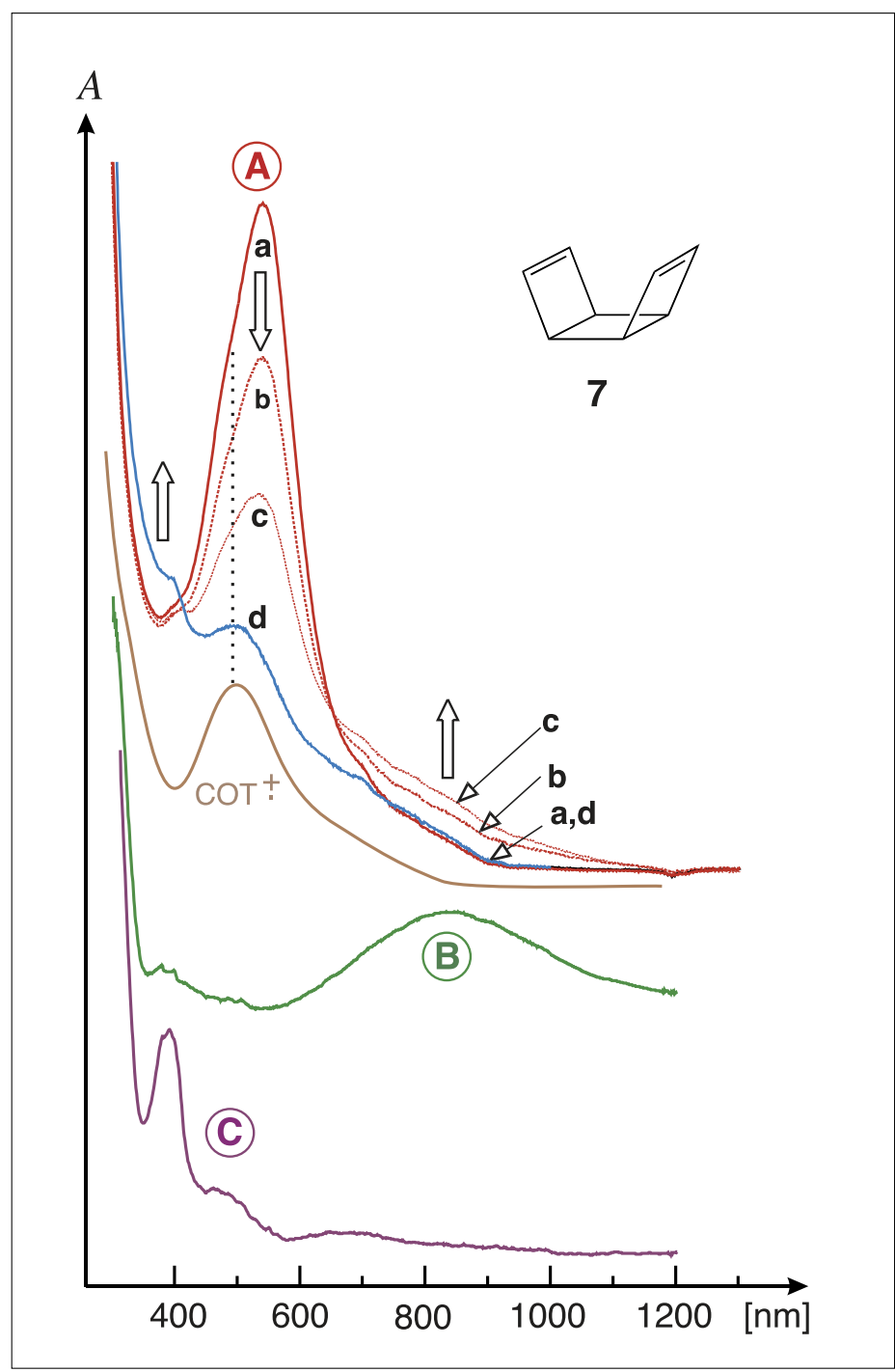




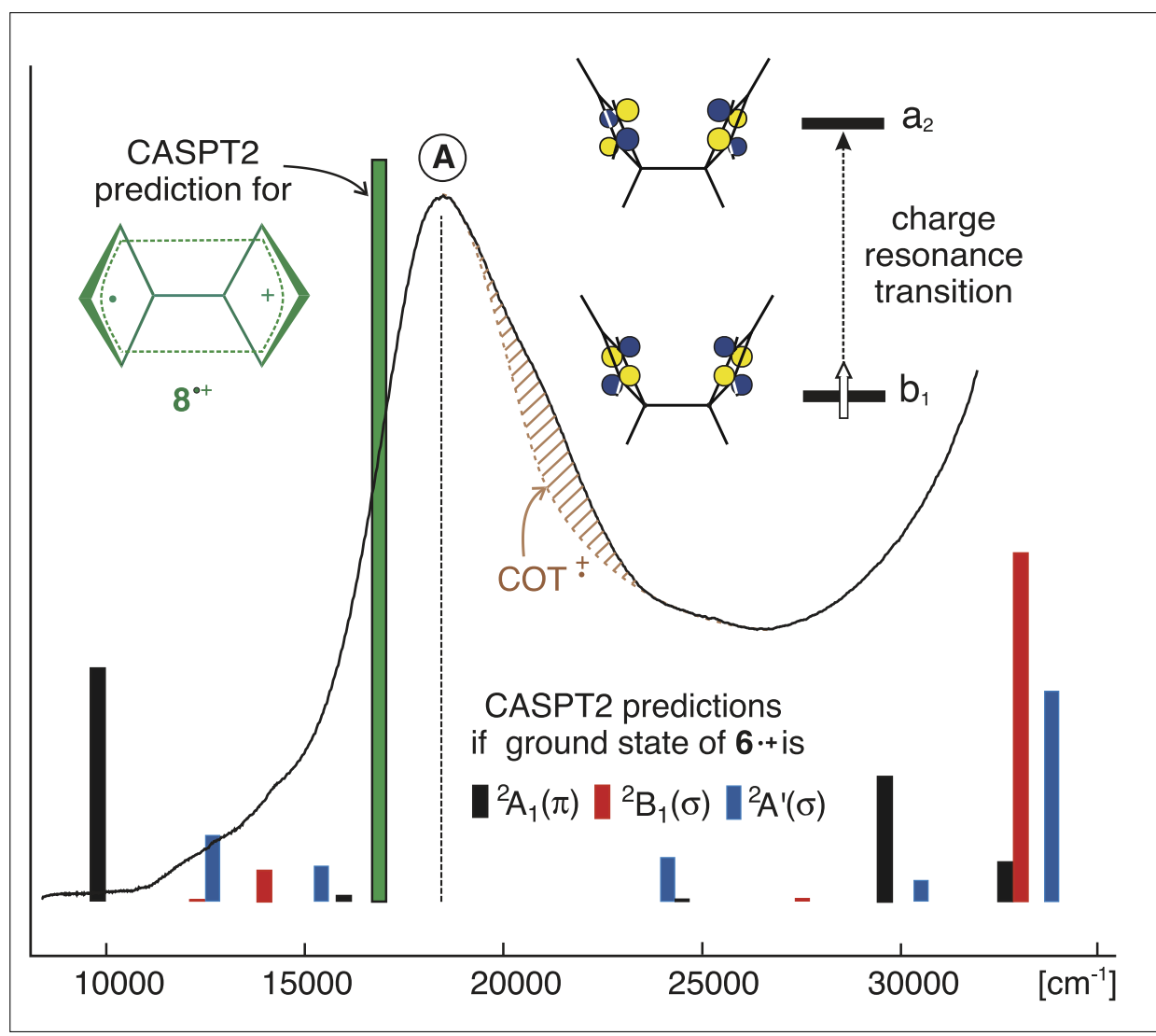

Fig. 3. Spectrum of species $A$ and CASSCF/CASPT2 predictions for the radical cation of 7 in its ${ }^{2} A_{1}$ (black bars), ${ }^{2} \mathrm{~B}_{1}$ (red bars), or ${ }^{2} \mathrm{~A}$ ' (blue bars) states (for details, see the original publication [6]). The green bar indicates the position of the strong charge-resonance transition of the bisallylic radical cation $\mathbf{8}^{\circ+}$.

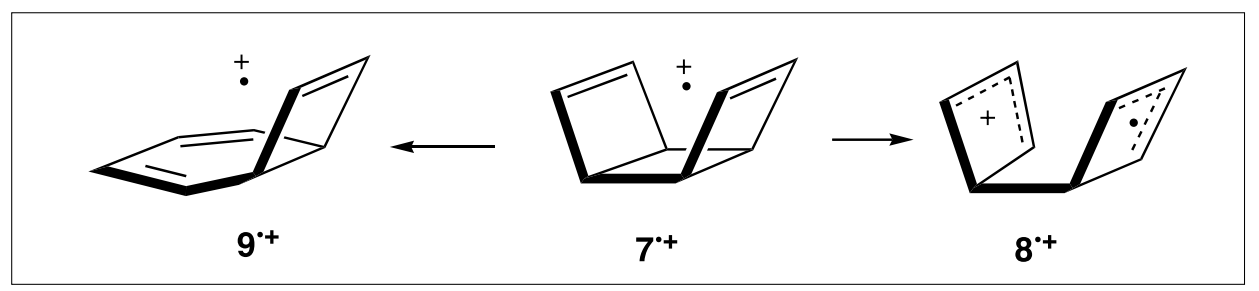

Scheme 3.

giving rise to a new band at $c a .400 \mathrm{~nm}$ (Fig. 2). Thus we are looking at a sequence of reactions of radical cations $\mathbf{A} \rightarrow \mathbf{B} \rightarrow \mathbf{C}$ that calls for assignments of the three species. Unfortunately it was impossible to follow this reaction in Ar matrices, so we had to forgo the use of IR spectroscopy to help in the process. Thus we had to rely solely on the UV/Vis spectra and consequently we needed a method that yields reliable predictions of such spectra.

At first we postulated that species A is the radical cation of 7. Examination of this hypothesis turned out to be rather complicated because $7^{\circ+}$ can be formed in three different close-lying electronic states, each of which was found to correspond to a minimum on the B3LYP/6-31G* potential surface (in fact, we later found that one of them serves as the direct precursor for $\mathrm{COT}^{*+}$ ). However, the electronic transitions computed for all three states by the mode for asymmetric deformation of the central four-membered ring that was associated with a suspiciously low frequency of only $154 \mathrm{~cm}^{-1}$. Following this mode led to a transition state a mere $0.2 \mathrm{~kJ} / \mathrm{mol}$ above the minimum, at the far side of which one bond of this four-membered ring undergoes cleavage to yield the bisallylic radical cation $\mathbf{8}^{\mathbf{+}}$ (Scheme 3). A CASPT2 calculation showed that this species has an excited state attainable in a very intense so-called 'charge resonance' transition [8] at $595 \mathrm{~nm}$ (green bar and inset in Fig. 3). The energy of this transition is very sensitive to the angle and the distance between the two cyclobutenylium moieties in $\mathbf{8}^{\circ+}$, hence a discrepancy of $0.2 \mathrm{eV}$ is well within the accuracy that can be expected from CASPT2 calculations, and the identity of species $\mathbf{A}$ was therefore established.

The assignment of the secondary product $\mathbf{B}$ turned out to be even more challenging, and this time density functional theory actually stood in the way of our finding the correct solution, as will be described below. Meanwhile we had, however, been able to identify the final product $\mathbf{C}$ as being identical to $\mathbf{9}^{\mathbf{}}$, the radical cation of bicyclo[4.2.0] octa-2,4,7-triene (Scheme 3), a simple valence isomer of $\mathbf{7}^{\bullet+}$ that would have been expected to arise in a single step from the latter, perhaps on the way to the radical cation of cyclooctatetraene. This finding made it very probable that the enigmatic species $\mathbf{B}$ must also be a valence isomer of $\mathbf{7}^{\mathbf{+}}$ and $\mathbf{9}^{\mathbf{}}$. As we had no clue what this species could be we engaged in a systematic search for $\mathrm{C}_{8} \mathrm{H}_{8}$ radical cations that might conceivably be formed from $7^{\circ+}$ and show a band at $820 \mathrm{~nm}$ with no further strong transitions in the visible.

The most promising candidate that emerged from this search was the tetracyclic radical cation $\mathbf{1 0}^{\circ+}$ for which CASPT2 predicted indeed a band of medium intensity at $820 \mathrm{~nm}$ followed only by some very weak transitions down to $300 \mathrm{~nm}$. To our surprise, $\mathbf{1 0}^{\circ+}$ turned out to be over $30 \mathrm{~kJ} /$ mol more stable than $\mathbf{8}^{\circ+}$ (at the $\operatorname{CCSD}(\mathrm{T}) /$ cc-pVDZ//B3LYP/6-31G* level) so a thermal rearrangement of $8^{\circ+}$ to $\mathbf{1 0}^{\circ+}$ appeared indeed feasible (Scheme 4). This reaction involves the formation of a new bond between the center of one of the cyclobutenyl moieties and the corner of the other, followed by the formation of a transannular ground states of $\mathbf{7}^{\mathbf{}}$ (the totally symmetric ${ }^{2} \mathrm{~A}_{1}$ state) by B3LYP turned up a normal
CASSCF/CASPT2 method are at odds with the observed $540 \mathrm{~nm}$ band (Fig. 3), which the conclusion that, on ionizat less rearrangement to the compound with spectrum A.

Computational quantum chemistry pointed us to the path that leads to the decay of $7^{\circ}+$. Analysis of the vibrational struc-

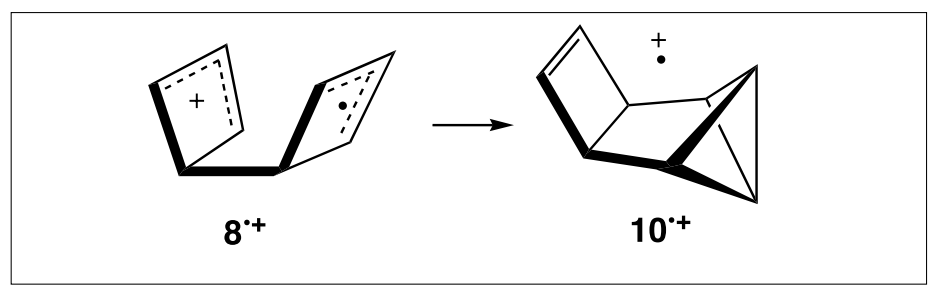

Scheme 4. 


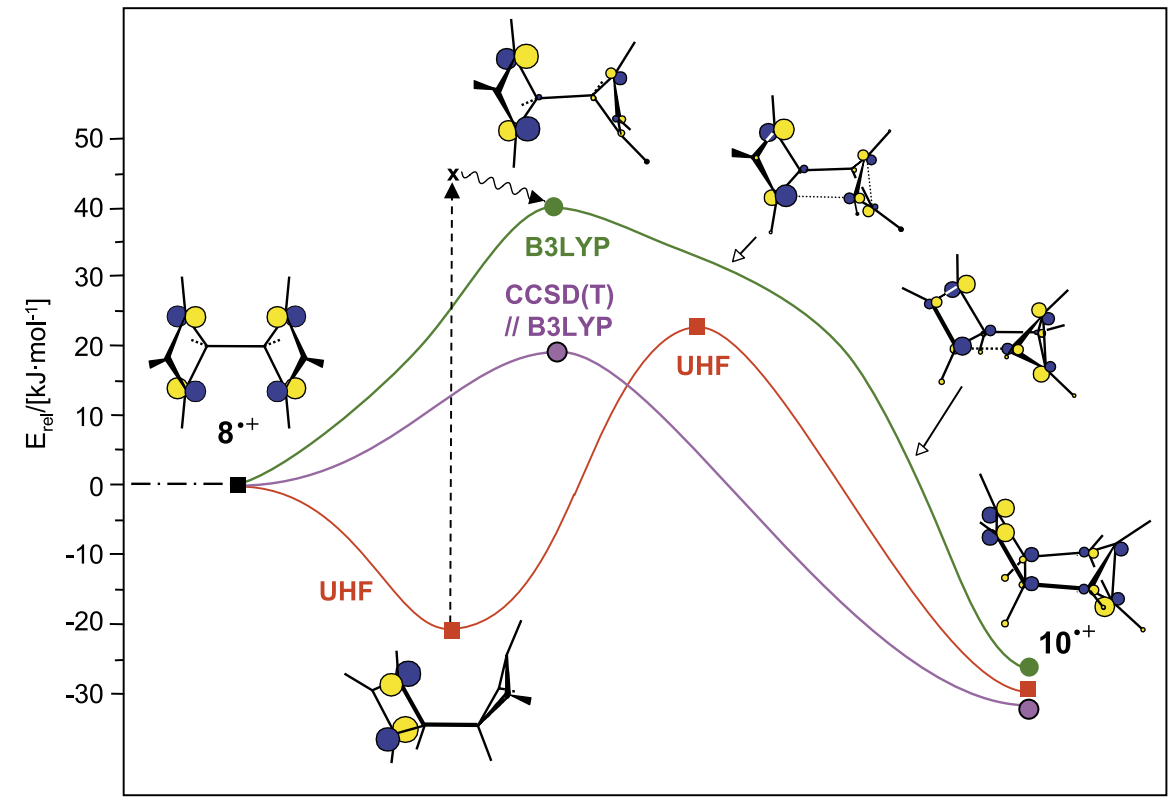

Fig. 4. Schematic representation of the potential surfaces for the rearrangement of $8^{\bullet+}$ to $10^{\circ+}$ by different methods. Note that the UHF method artefactually localizes spin and charge in opposite halves of $\mathbf{8}^{\mathbf{*}}$, whereas UB3LYP shows an artefactual destabilization of this structure. The insets show the change in shape of the singly occupied Kohn-Sham MO along the UB3LYP reaction coordinate.

bond in the first cyclobutenyl ring, as depicted in Fig. 4.

However, the mapping of this reaction path turned out to be impossible by DFT because the formation of the new bond requires a preceding localization of spin and charge in opposing cyclobutenium moieties of $\mathbf{8}^{\cdot+}$. Such processes cannot be modeled correctly by current DFT methods [9], because the inherently incomplete cancellation of the electron self repulsion in exchange functionals results in an artefactual overstabilization of delocalized $v s$. localized structures which effectively prevents such localizations from taking place in radical cations, even in cases where they should [10][11].

Conversely, at the UHF level (which ignores the dynamic electron correlation effects that result in the stabilization of structures with delocalized electrons) $\mathbf{8}^{\bullet+}$ relaxes spontaneously to a structure with localized spin and charge (Fig. 4), in disagreement with experiment, because such a localized structure of $\mathbf{8}^{\mathbf{}}$ would not show an intense charge resonance band. At this level of theory, it is then easy to find a transition state for the formation of the new bond on the way to $\mathbf{1 0}^{\mathbf{}}$, but the corresponding activation energy is of course not realistic. Eventually we succeeded in locating this transition state also on the B3LYP potential surface, and single-point CCSD(T)/cc-pVDZ calculations at the B3LYP stationary points resulted in an activation enthalpy of only 18 $\mathrm{kJ} / \mathrm{mol}$ for the $\mathbf{8}^{++} \rightarrow \mathbf{1 0}^{\bullet+}$ rearrangement.
Experiment seems to indicate that the barrier for this process, which occurs within minutes at $75 \mathrm{~K}$, is even lower. At $90 \mathrm{~K}$, $\mathbf{1 0}^{\circ+}$ finally rearranges to $\mathbf{9}^{\circ+}$ (which can also be formed by photolysis of $\mathbf{8}^{\mathbf{}}$ ) over a computed barrier of $56 \mathrm{~kJ} / \mathrm{mol}$.

An interesting question that was raised by the above observations was, why $7^{+}$, which we had expected to be a perfectly stable radical cation, spontaneously decays to $\mathbf{8}^{\bullet+}$. The answer is that the presence of low-lying electronic states of different symmetry than the ground state may cause substantial flattening of the ground-state potential surface, or even fect known as vibronic coupling [12][13]. The size of this effect is proportional to the size of the so-called derivative coupling integral

$$
f_{Q}^{I J}(\boldsymbol{R})=\left\langle\Psi_{I}(\boldsymbol{r}, \boldsymbol{R})\left|\frac{\partial}{\partial Q}\right| \Psi_{J}(\boldsymbol{r}, \boldsymbol{R})\right\rangle_{\boldsymbol{r}}
$$

where $Q$ represents a non-totally symmetric distortion which causes the wavefunctions $\Psi_{\mathrm{I}}$ and $\Psi_{\mathrm{J}}$ (which are of different symmetry at a totally symmetric geometry $R_{0}$ ) to mix, and indirectly proportional to the energy difference $E_{\mathrm{I}}-E_{\mathrm{J}}$ between the two interacting states. Both quantities can be computed, and such calculations may allow predictions to be made about the way in which a species spontaneous distortions, through an ef- might undergo facile or even spontaneous distortion.

As an example Fig. 5 shows the derivative coupling vectors (which indicate the distortion along which maximal mixing between two wavefunction occurs, and how strong that mixing will be) for the coupling of the ${ }^{2} \mathrm{~A}_{1}$ ground state of $\mathbf{7}^{\bullet+}$ with two excited states, ${ }^{2} \mathrm{~B}_{1}$ which lies $1.21 \mathrm{eV}$, and ${ }^{2} \mathrm{~B}_{2}$ which lies $1.98 \mathrm{eV}$ above the ground state. The extent of coupling is similar in both cases, but it is the distortion that is caused by mixing with the higher-lying ${ }^{2} \mathrm{~B}_{2}$ state which 'drives' the decay of $7^{\circ+}$ to $\mathbf{8}^{\cdot+}$. This finding leads to another, often neglected factor: what will be the effect of vibronic mixing, which depends not only on the magnitude of the coupling between two states, and by their separation, but also on the force constant which opposes distortion. In the first case the distortion involves stretching and compressing $\mathrm{C}=\mathrm{C}$ double bonds, hence the force constant is high, whereas in the second case, more easily deformed single bonds are involved in the distortion.

In fact, our research has led us to the conclusion that the often surprisingly facile rearrangements of radical ions are invariably dictated by vibronic coupling, and we have since then discovered several more cases where their reactivity can be rationalized in such terms [14-16]. A very intriguing case that kept us busy for several years concerned the fate of the highly strained hydrocarbon, [1.1.1]propellane (11) on ionization [17]. This compound has a totally symmetric, almost nonbonding HOMO, thus ionization was not expected to lead to much structural change. However, under the influence of higher-lying degenerate states the radical cation of [1.1.1] propellane undergoes what is appropriately called a second-order Jahn-Teller distortion which projects the compound onto a potential energy chute that does not end until three lateral bonds have been broken and an interesting isomeric species, the dimethylene allene radical cation $\left(\mathbf{1 2}^{\mathbf{}}+\right)$ is formed (Scheme 5).

\section{Thermoreversible Photocyclization of a Pyrazolotriazole}

The third and final example of our work is another case where a judicious combination of experiment and theory led to the solution of a puzzling question [18]: In 1991, Albini et al. had observed that the pyrazolo[1,2-a]benzotriazoles 13a,b undergo a thermoreversible photoreaction to products which they tentatively identified as the nitrenes $\mathbf{1 4 a}, \mathbf{b}$ which are thought to be the direct precursors of 13a,b obtained 

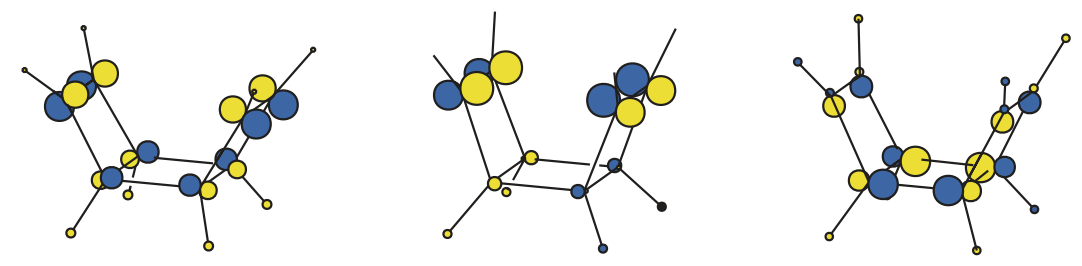

$\operatorname{SOMO} a_{1}\left(\pi_{+}\right)$

$\mathrm{b}_{1}\left(\pi_{-}\right)$

$\mathrm{b}_{2}(\sigma)$
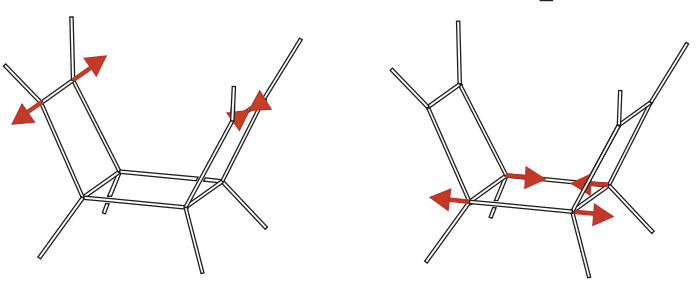

${ }^{2} \mathrm{~A}_{1}$ and ${ }^{2} \mathrm{~B}_{1}$

${ }^{2} \mathrm{~A}_{1}$ and ${ }^{2} \mathrm{~B}_{2}$

CASPT2 energy difference:

\section{$1.98 \mathrm{eV}$}

Fig. 5. Singly occupied MOs in different states of $\mathbf{7}^{\bullet+}$ and derivative coupling vectors indicating the direction of maximal interaction between the ${ }^{2} \mathrm{~A}_{1}$ ground state and the ${ }^{2} \mathrm{~B}_{1}$ and ${ }^{2} \mathrm{~B}_{2}$ excited states of $7^{\bullet+}$.

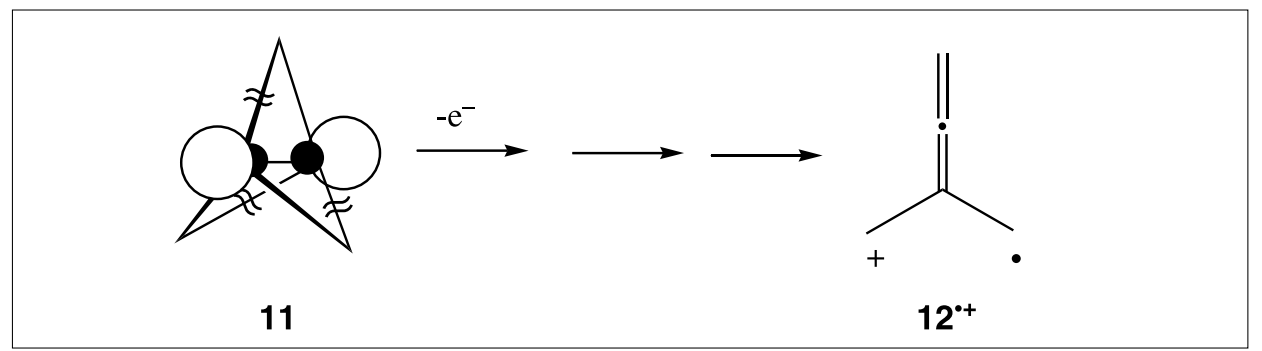

Scheme 5 .

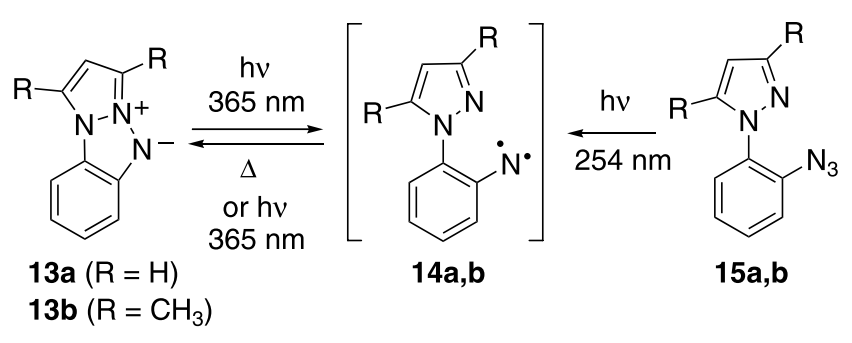

Scheme 6 .

by photolysis of the corresponding azides 15 [19] (Scheme 6).

However, this assignment appeared untenable because (a) singlet arylnitrenes have generally much shorter lifetimes than those observed for the photoproducts of compounds 13, and (b) triplet nitrenes 14 are not expected to revert thermally to $\mathbf{1 3}$. We reckoned that, if we could generate the metastable photoproduct of compounds 13 under conditions where it would be long-lived, we might be able to identify it spectroscopically. Indeed we found that in Ar matrices the photoproduct is indefinitely stable but can be partially reverted

to 13 by irradiation at $313 \mathrm{~nm}$. This allowed us to record the UV/VIS and IR spectra of this enigmatic species. The UV-spectra (Fig. 6) indicated the absence of a highly conjugated chromophore but otherwise contained no useful information, while the rich IR spectra showed no bands that were indicative of any particular chromophore.

As we did not know how to interpret our experimental findings we turned to theory, asking what could be possible photoproducts of $\mathbf{1 3}$ that would revert thermally to that compound. Thereby we ran into a curious problem which sidetracked us for a while, namely that the identity of the first excited state depended strongly on the way we ran the CASSCF calculations on 13, in particular whether we used state-averaging or not. Actually this problem (which has not been solved to date!) prevented us from assigning the spectrum of $\mathbf{1 3}$ shown in Fig. 6 on the basis of CASSCF/CASPT2 calculations. However, since a variety of other electronic structure calculations agreed in predicting that the first excited state of 13 (which gives rise to the intense structured $300-370 \mathrm{~nm}$ band) is dominated by $\mathrm{HOMO} \rightarrow$ LUMO excitation, we eventually assumed that the observed photoreaction originates from this state.

Because the two frontier orbitals of $\mathbf{1 3}$ are localized essentially in the heterocyclic part, we decided to model the photoreactivity on the basis of compound $\mathbf{1 6}$ which does not contain the fused benzene ring. There (as in 13) the HOMO is $\pi$-antibonding between the two allylic moieties making up the pyrazolopyrazole, whereas the LUMO is bonding (Fig. 7, upper left), which suggests that the first excited state may be prone to formation of a $\sigma$-bond between the allylic termini. Indeed, CIS geometry optimization of this state led to spontaneous cyclization to a hetero-semibullvalene 17 which reverts to $\mathbf{1 3}$ over a (B3LYP/6-312G*) barrier of ca. $13 \mathrm{kcal} /$ mol. In 17 the HOMO is bonding and the LUMO is antibonding along the newly formed bond, hence HOMO $\rightarrow$ LUMO excitation is expected to result in cleavage of that bond, i.e. return to 13. This feature was also borne out by CIS optimization of $\mathbf{1 7}$ in its first excited state.

In a two-dimensional representation (Fig. 7) the two photoreaction paths cross which suggests that they might share a conical intersection [20]. Indeed we were able to locate (by state-averaged CASSCF calculations) this 'funnel' through which both excited state species pass on their way to the ground state, and it was found to lie close to the transition state for the thermal reaction. Theory says that, after passing through the conical intersection, systems may evolve in first order along two distinct directions which are represented by a pair of orthogonal vectors. One of these is the derivative coupling vector that we have met in Section 3 (Fig. 5 ) while the other is called the gradient difference vector

$$
\Delta \mathbf{g}^{I J}=\frac{\partial\left(E_{J}-E_{I}\right)}{\partial \mathbf{Q}}
$$

which indicates the direction along which the difference between the gradients on the excited and the ground state potential surface is maximal. In the case of the conical 
Fig. 6. Spectrum of pyrazolotriazole 13a in an Ar matrix at 12 $\mathrm{K}$ (red line), and after bleaching at $365 \mathrm{~nm}$ (blue line). The dotted line indicates the partial recovery of 13a on bleaching the photoproduct $\mathbf{X}$ at 313 $\mathrm{nm}$ (probably because a photostationary equilibrium is reached).

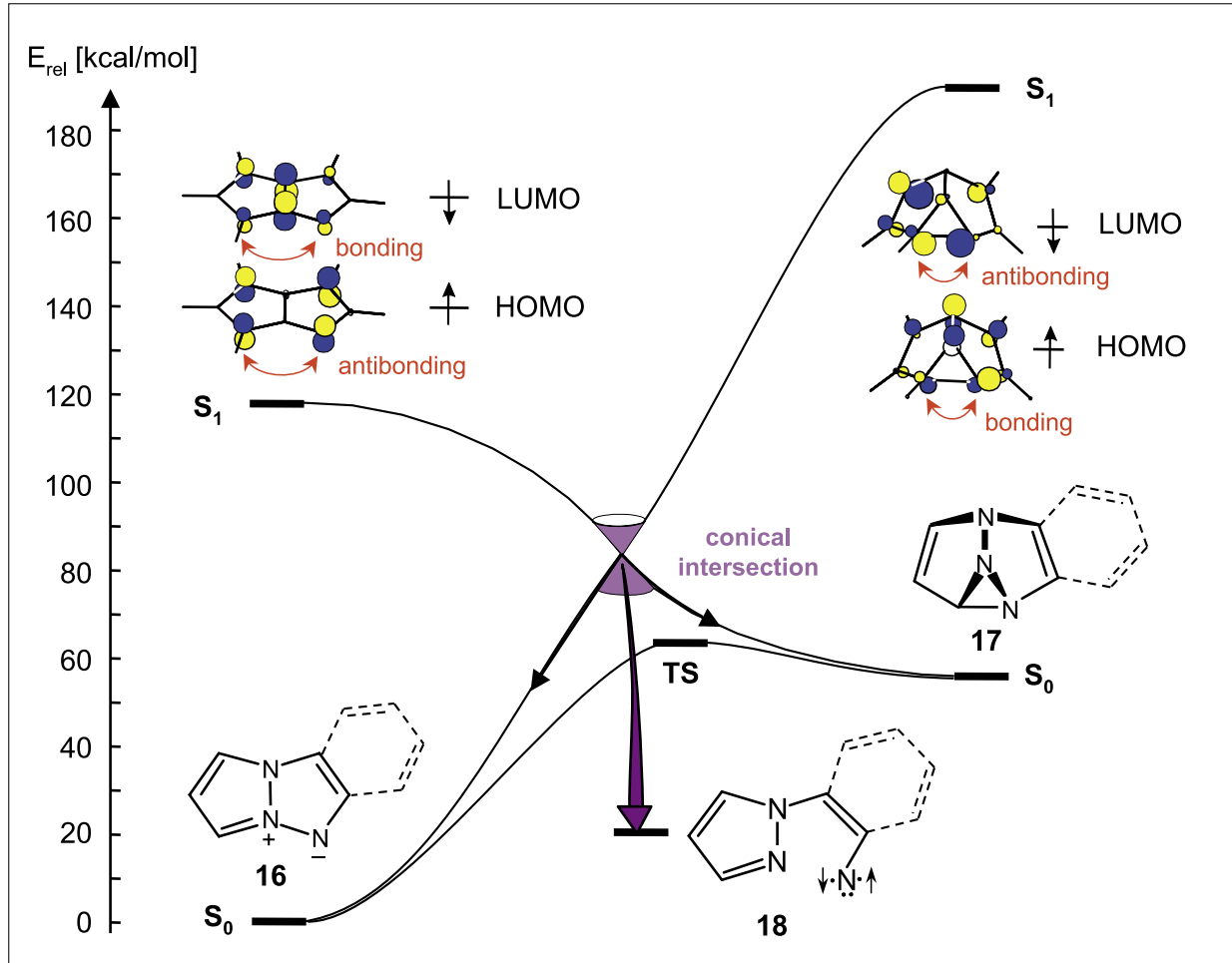

Fig. 7. Schematic representation of the ground- and excited-state potential surfaces on which the interconversion between $\mathbf{1 6}$ and $\mathbf{1 7}$ takes place. The position of the annulated benzene ring in compounds 13 and 19, respectively, is indicated with dashed lines. Explanation, see text.

intersection between $\mathbf{1 6}$ and $\mathbf{1 7}$ the derivative coupling vector turned out to coincide roughly with the direction of negative curvature at the thermal transition state, i.e. with the reaction coordinate, whereas distortion along the gradient difference vector led to a cleavage of the $\mathrm{N}-\mathrm{N}$ bond and, on geometry optimization, to formation of the vinylnitrene 18.

We then went on to apply the structural hypothesis which our computer experiments had provided to the 'full' system 13, and were happy to find that the resulting compounds 19 (Scheme 7) are fully com- patible with the UV/VIS and IR-spectra of the photoproducts we had observed in our matrix experiments.

Eventually, we discovered that 19a persists for some time at $-80{ }^{\circ} \mathrm{C}$ in methylene chloride which allowed us to measure its NMR spectra which also turned out to be in full accord with the triazasemibullvalene structure (in particular, it showed a very telling ${ }^{13} \mathrm{C}$ resonance at $80 \mathrm{ppm}$ which clearly indicated a saturated carbon atom). In contrast, $\mathbf{1 3 b}$ appeared to be photostable under the same conditions and was only slowly degraded in an irreversible process. This finding agrees also with calculations which predict that the barrier for the decay $\mathbf{1 9} \rightarrow \mathbf{1 3}$ is about $3.4 \mathrm{kcal} / \mathrm{mol}$ lower in the dimethyl derivative which apparently does not persist at $-80^{\circ} \mathrm{C}$.

Upon prolonged irradiation compounds 13 are eventually degraded to polymers. The reason for this is probably that each time the molecules pass through the conical intersection, some of them are diverted to the phenylnitrenes $\mathbf{1 4}$ (the analogon of the vinylnitrene 18 in Fig. 7) which then relax to their triplet ground state where they are quenched by oxygen and other species. The same mechanism is probably also responsible for the broadening of the NMRbands on photolysis of 13b in solution due to the presence of the paramagnetic triplet state of 14.

\section{Conclusion}

In this brief account three examples from our recent work were chosen to illustrate the role that computational quantum chemistry plays in our program of studies on reactive intermediates. Indeed, without the impressive progress in hardware as well as quantum chemical software and methodology that we have witnessed during the past 15 years, much of our experimental observations would have remained unexplained, and our understanding of the reactivity of radical cations and other reactive intermediates would have remained as rudimentary as it was when we started this research.

Conversely, each time we decide to carry out quantum chemical calculations, we do this with the goal of finding answers to questions that arise through our own experimental work. Much of this work deals with species that have a somewhat peculiar electronic structure (e.g. conjugated radicals, compounds with open-shell singlet ground states etc.) and are therefore not always amenable to treatment by standard methods. This occasionally leads us to interesting discoveries, such as the failure of DFT to properly describe radical ions where spin and/or charge are localized in 


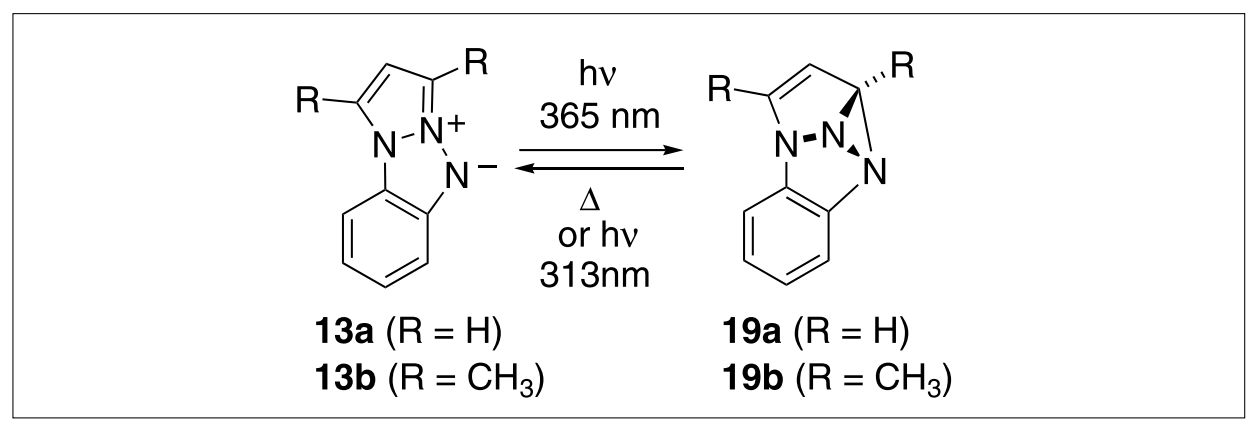

Scheme 7.

different but structurally equivalent moieties (Fig. 4) [9].

To sum up, it is through the interplay and the exploitation of synergisms between experiment and theory that we have been able to address a number of interesting and fundamental questions in the field of reactive intermediates; questions that neither experiment nor theory alone could have solved. An added benefit of this activity is that students get a dual training which they were often able to exploit in their own careers.

\section{Acknowledgements}

Above all I would like to express my gratitude to Prof. Edwin Haselbach who introduced me to the exciting field of radical ions and to semiempirical quantum chemistry during my graduate work in Basel, and who provided the opportunity for me to start my own career in Fribourg. Secondly, I owe much to the graduate students who, by their readiness to confront the double challenge of learning how to master the experimental techniques and the different quantum chemical programs in use in our laboratory, as well as by their tenacity and perseverance in the face of tough problems, have made it possible for us to find our way through several mazes which led us to rewarding scientific insight (and a PhD thesis). Finally my thanks go to my colleagues from here and abroad with whom we have collaborated over the course of time and who were kind enough to offer us patient education on subjects where they are experts.

Received: May 19, 2005
[1] T. Bally, in 'Reactive Intermediate Chemistry', Eds. R.A. Moss, M.A. Platz, M. Jones, Wiley \& Sons, New York, 2004, Chapter 17, p. 797.

[2] Z. Zhu, T. Bally, L.L. Stracener, R.J. Mc2863.

[3] C.M. Geise, Y. Wang, O. Mykhaylova, B.T. Fink, J.P. Toscano, C.M. Hadad, J. Org. Chem. 2002, 67, 3079.

[4] Y.Wang, C.M. Hadad, J.P. Toscano, J. Am. Chem. Soc. 2002, 124, 1761.

[5] K. Andersson, B.O. Roos, in 'Modern Electronic Structure Theory', World Scientific Publ. Co., Singapore, 1995, Part 1, Vol. 2, p 55.

[6] T. Bally, S. Bernhard, S. Matzinger, J.-L. Roulin, G.N. Sastry, L. Truttmann, Z. Zhu, A. Marcinek, J. Adamus, R. Kaminski, J. Gebicki, F. Williams, G.-F. Chen, M.P. Fülscher, Chem. Eur. J. 2000, 6, 858.

[7] T. Bally, L. Truttmann, S. Dai, F. Williams, J. Am. Chem. Soc. 1995, 117, 7916.

[8] B. Badger, B. Brocklehurst, Nature (London) 1968, 219, 263.

[9] T. Bally, G.N. Sastry, J. Phys. Chem. A 1997, 101, 7923.

[10] M. Sodupe, J. Bertran, L. Rodriguez-Santiago, E.J. Baerends, J. Phys. Chem. A 1999, 103, 166.

[11] B. Braïda, P.C. Hiberty, A. Savin, J. Phys. Chem. A 1998, 102, 7872. Vibronic Interactions in Modern Chemistry', Plenum Press, New York, 1983. Mahon, J. Am. Chem. Soc. 1999, 121

[12] I.B. Bersuker, 'The Jahn-Teller Effect and
[13] H. Köppel, L.S. Cederbaum, W. Domcke, S.S. Shaik, Angew. Chem. 1983, 95, 221.

[14] K. Schroeter, D. Schröder, H. Schwarz, G.D. Reddy, O. Wiest, C. Carra, T. Bally, Chem. Eur. J. 2000, 6, 4422.

[15] B. Müller, T. Bally, F. Gerson, A. de Meijere, M.von Seebach, J. Am. Chem. Soc. 2003, 125, 13776.

[16] T. Bally, A. Maltsev, F. Gerson, D. Frank, A. de Meijere, J. Am. Chem. Soc. 2005, 127, 1983.

[17] F. Williams, R. Pappas, B. Müller, T. Bally, manuscript in preparation, 2005.

[18] C. Carra, T. Bally, T.A. Jenny, A. Albini, Photochem. Photobiol. Sci. 2002, 1, 38.

[19] A. Albini, G. Bettinetti, A. Minoli, J. Am. Chem. Soc. 1991, 113, 6928.

[20] 'Conical Intersections', Eds. W. Domcke, D.R. Yarkony, H. Köppel, World Scientific Publ., Singapore, 2004. 